\title{
Outcomes of simultaneous vs. sequential pars plana vitrectomy and cataract surgery
}

\author{
ANDREEA DANA MORARU ${ }^{1,2^{*}}$, DĂNUȚ $\operatorname{COSTIN}^{1,2}$, \\ RADU LUCIAN MORARU ${ }^{3 *}$ and DANIEL CONSTANTIN BRANISTEANU ${ }^{1,4}$
}

\author{
${ }^{1}$ Department of Ophthalmology, ‘Grigore T. Popa' University of Medicine and Pharmacy, 700115 Iași; \\ ${ }^{2}$ Department of Ophthalmology, 'N. Oblu' Clinical Hospital, 700309 Iași; ${ }^{3}$ Department of Otorhinolaryngology, \\ 'Transmed Expert' Medical Center, 700011 Iași; ${ }^{4}$ 'Retina Center' Eye Clinic, 700126 Iași, Romania
}

Received June 5, 2020; Accepted July 6, 2020

DOI: $10.3892 / \mathrm{etm} .2020 .9313$

\begin{abstract}
The coexistence of cataract and vitreoretinal diseases is common in clinical practice. The technological achievements of the last decade in both types of surgeries, allow simultaneous interventions on lens and retina. This retrospective study assessed the morphofunctional results obtained after performing simultaneous surgeries in a series of 87 patients presenting with both pathologies. The cases were retrospectively reviewed from their clinical records from 2017 to 2019. The patients were divided into two groups: group 1 comprising 41 cases underwent the combined procedure and group 2 comprising 46 patients underwent vitrectomy, followed by cataract surgery after 3-10 months. The functional and anatomical outcomes and the complications were assessed pre- and post-operatively. The statistical analysis was carried out by MaxStat software. The final best corrected visual acuity (BCVA), in group 1 was $<0.1$ in $4.88 \%$ of the cases, 0.1 to 0.3 in $17.07 \%, 0.3$ to 0.5 in $51.22 \%$ and $>0.5$ in $26.83 \%$ eyes. In group 2, the final BCVA, after both surgeries were completed was $<0.1$ in $8.7 \%$ cases, 0.1 to 0.3 in $30.43 \%, 41.3 \%$ were 0.3 to 0.5 and $19.57 \%$ were $>0.5$. During the cataract surgery, the most common intraoperative complication was miosis, followed by posterior capsule rupture. Postoperative, $12.2 \%$ cases from group 1 developed an immediate inflammatory reaction in the anterior chamber, and $17.07 \%$ presented with posterior capsular opacification (PCO). Simultaneous surgery is safe and effective in obtaining a good morphological and functional result and offers the advantage of a clear eye media that allows a safer vitrectomy and thus a
\end{abstract}

Correspondence to: Professor Dănuţ Costin, Department of Ophthalmology, 'Grigore T. Popa' University of Medicine and Pharmacy, 16 University Street, 700115 Iași, Romania

E-mail: oftalmoconsultiasi@yahoo.ro

${ }^{*}$ Contributed equally

Key words: cataract, vitrectomy, simultaneous surgery, combined procedure, sequential surgery quicker rehabilitation of the patient. The analysis of the risks and benefits of each procedure should be taken into account and the cases selected individually for either simultaneous or sequential surgery in order to obtain the best outcomes.

\section{Introduction}

Recent advances in both cataract and posterior eye segment surgery allow a larger number of complex cases to be operated in a single procedure. Cataract surgery is the most common ophthalmological surgical procedure performed worldwide and vitreoretinal surgery is the third (1).

The coexistence of cataract and vitreoretinal diseases is common, not only in elderly patients but also after eye trauma, uveitis and diabetes. The presence of both disorders in the same eye renders the case to be surgically challenging and the evolution to be somewhat unexpected. A dense cataract in an eye with vitreoretinal pathology requiring surgical intervention can make a routine surgery difficult and raise the intra and postoperative rate of adverse reactions. On the other hand, it is a known fact that cataract progresses after posterior eye segment surgery.

The technological achievements of the last decade in both types of surgeries, regarding microincisional machinery and instrumentation, allow simultaneous interventions on both lens and retina, thus achieving a quicker recovery of the patient and a higher effectiveness rate of the surgeon. As both medical and economic factors influence the decision of the surgeon in choosing the appropriate procedure for the patient, the current trend in Europe is to perform a combined procedure for cases presenting with both pathologies, as opposed to US surgeons. Presumably, the combined procedure is prone to an increased rate of postoperative complications such as intraocular inflammation and increase in intraocular pressure (IOP) (2). Nevertheless, when sequential surgery is chosen and phacoemulsification is performed after pars plana vitrectomy (PPV), the cataract extraction intervention is more difficult due to either a harder nucleus or due to different morphological conditions in a vitrectomized or silicone oil-filled eye.

The present study assessed the morphofunctional results obtained after performing combined phacoemulsification and vitrectomy in a series of patients presenting both pathologies. 


\section{Patients and methods}

A series of 87 cases, presenting with cataract and vitreoretinal pathology, were retrospectively reviewed from their clinical records from 2017 to 2019. The study was approved by the Ethics Committee of 'N. Oblu' Clinical Hospital (Iași, Romania) and informed consent was obtained from each patient. The patients included in the study were diagnosed with the following vitreoretinal disorders: Rhegmatogenous retinal detachment, vitreous hemorrhage from proliferative diabetic retinopathy or blunt trauma, tractional retinal detachment all associated with cataract. Cases presenting with penetrating trauma were excluded from the study. The patients were divided into two groups: group 1 comprising 41 cases that underwent the combined procedure and group 2 comprising 46 patients that underwent PPV for the vitreoretinal pathology, followed by cataract surgery after a period of time between 3 and 10 months.

The visual acuity (VA), IOP, the anatomical outcome and the complications were assessed preoperative and postoperative. The VA was tested on Snellen chart. The IOP was measured by Goldmann tonometry. Both the anterior and the posterior segment of the patients' eyes were examined at the slit lamp before surgery and in the first and third day postoperative. After discharge, the cases returned for follow-up at 2 weeks, and at 1,3 and 6 months.

The intraocular lens (IOL) power was assessed by optical biometry, or in case of dense cataracts by ultrasound biometry, using the SRK-T formula. An ultrasound of the posterior segment of the eye was performed in cases of dense cataracts.

The surgical procedure consisted of phacoemulsification of the lens by clear corneal incisions and 23 or $25 \mathrm{G} \mathrm{PPV}$, under peribulbar anesthesia, after obtaining the informed consent of the patient. In cases that underwent the combined procedure, the trocars were put in place before the cataract extraction. The phacoemulsification was performed using one $2.2 \mathrm{~mm}$ main incision and two $1.2 \mathrm{~mm}$ sideports, under the protection of one cohesive and one dispersive ophthalmic viscosurgical device (OVD). A hydrophobic, foldable, acrylic IOL was placed in the capsular bag or ciliary sulcus at the end of the cataract surgery. If phacoemulsification and PPV were performed simultaneously, the main corneal incision was secured by a 10.0 nylon suture during vitrectomy. In all cases requiring endotamponade, $1000 \mathrm{cS}$ or $5000 \mathrm{cS}$ silicone oil was used.

In the postoperative period, the patients were prescribed topical antibiotic and steroidal anti-inflammatory drugs 5 times a day for 1 week and tapered until the end of the first month. A few cases needed cycloplegic therapy with topical mydriatics 2-3 times a day for 1 week.

The statistical analysis was done using MaxStat software and the data were expressed as either percentage or mean values. $\mathrm{P}<0.05$ was considered statistically significant.

\section{Results}

Eighty-seven eyes from 87 patients were included in the study. Group 1, which underwent combined procedure was comprised of $27(65.85 \%)$ women and 14 (34.15\%) men and group 2, in which the surgical procedures were performed separately, consisted of 28 (60.87\%) women and 18 (39.13\%) men. There was no significant difference between the mean age of the groups: $58 \pm 9.42$ in group 1 and $56 \pm 11.3$ in group 2 .

In group 1 there were $12(29.27 \%)$ patients diagnosed with rhegmatogenous retinal detachment, 6 (14.64\%) with tractional retinal detachment and $23(56.09 \%)$ with vitreous hemorrhage. In group 2, $24(52.17 \%)$ patients were diagnosed with rhegmatogenous retinal detachment, 3 (6.53\%) with tractional retinal detachment and $19(41.3 \%)$ with vitreous hemorrhage.

The preoperative best corrected visual acuity (BCVA) did not differ significantly between the two groups, $32(78.05 \%)$ patients from group 1 had a VA $<0.1 ; 7$ (17.07\%) had a VA between 0.1 and 0.2 and $2(4.88 \%)$ had a VA of 0.3 . In group 2, a VA $<0.1$ was recorded in 29 (63.05\%) patients, VA between 0.1 and 0.2 in $12(26.09 \%)$ patients and a VA between 0.3 and 0.4 in $5(10.86 \%)$ patients. The final BCVA, after surgery, in group 1 was $<0.1$ in $3(7.32 \%)$ patients, between 0.1 and 0.3 in $6(14.63 \%)$ patients, between 0.3 and 0.5 in $21(51.22 \%)$ patients and $>0.5$ in $11(26.83 \%)$ patients. In group 2, the final BCVA, after both surgeries were completed was $<0.1$ in $4(8.7 \%)$ patients, between 0.1 and 0.3 in $14(30.43 \%)$ patients, between 0.3 and 0.5 in $19(41.3 \%)$ patients and $>0.5$ in $9(19.57 \%)$ patients. The final BCVA did not differ significantly between the two groups, but there was a larger percentage of eyes with a postoperative VA comprised between 0.1 and 0.3 , in group 2 (30.43\%), comparative to group $1(14.63 \%)$ and more eyes from group 1 (51.22\%) had a VA of $0.3-0.5$ by comparison to $41.3 \%$ in group 2 . The simultaneous surgery group had a higher rate of eyes that benefited from a final BCVA of $>0.3(78.05 \%)$, by comparison to the sequential surgery group, in which $60.87 \%$ obtained a postoperative VA of $>0.3$.

Similar values of the IOP were registered in both groups preoperative. In group 1 the mean IOP was $14 \pm 6.3 \mathrm{mmHg}$ and in group 2 the mean IOP was $13 \pm 7.1 \mathrm{mmHg}$. At the end of the first month of postoperative follow-up, the mean IOP in group 1 was $18 \pm 6.4 \mathrm{mmHg}$ and in group 2 was $15 \pm 8.5 \mathrm{mmHg}$. However, at the end of the first year of follow-up, the mean IOP had similar values in both groups: $13 \pm 5.1 \mathrm{mmHg}$ in group 1 and $14 \pm 6.8 \mathrm{mmHg}$ in group 2. One patient from group $1(2.4 \%)$ and $3(6.5 \%)$ patients from group 2 required topical hypotensive treatment during the whole follow-up period.

During the cataract surgery, the most common intraoperative complication was miosis present in $9(21.95 \%)$ eyes from group 1 and 12 eyes (26.08\%) from group 2, followed by posterior capsule rupture in 3 eyes $(7.31 \%)$ from group 1 and 7 eyes $(15.21 \%)$ in group 2, $1(2.4 \%)$ hemorrhage in the anterior chamber (AC) in group 1 and 4 eyes $(8.7 \%)$ with diminished AC depth from group 2, due to pressure exerted by the silicone oil on the posterior capsule. During PPV the most common intraoperative complication encountered was the difficulty of shaving the vitreous base in 24 eyes $(52.17 \%)$ with un-operated cataract from group 2 and the difficulty of visualization of the vitreous base in 13 eyes (31.7\%) with IOL from group 1. There were no iatrogenic tears registered in either group.

During the follow-up period, 15 eyes (32.6\%) from group 2 registered a significant advance in cataract density and had to be operated in the third month after PPV. The rest of the eyes from group 2 benefited from cataract surgery after a period of 3-6 months after PPV - 18 eyes (39.13\%) and after 


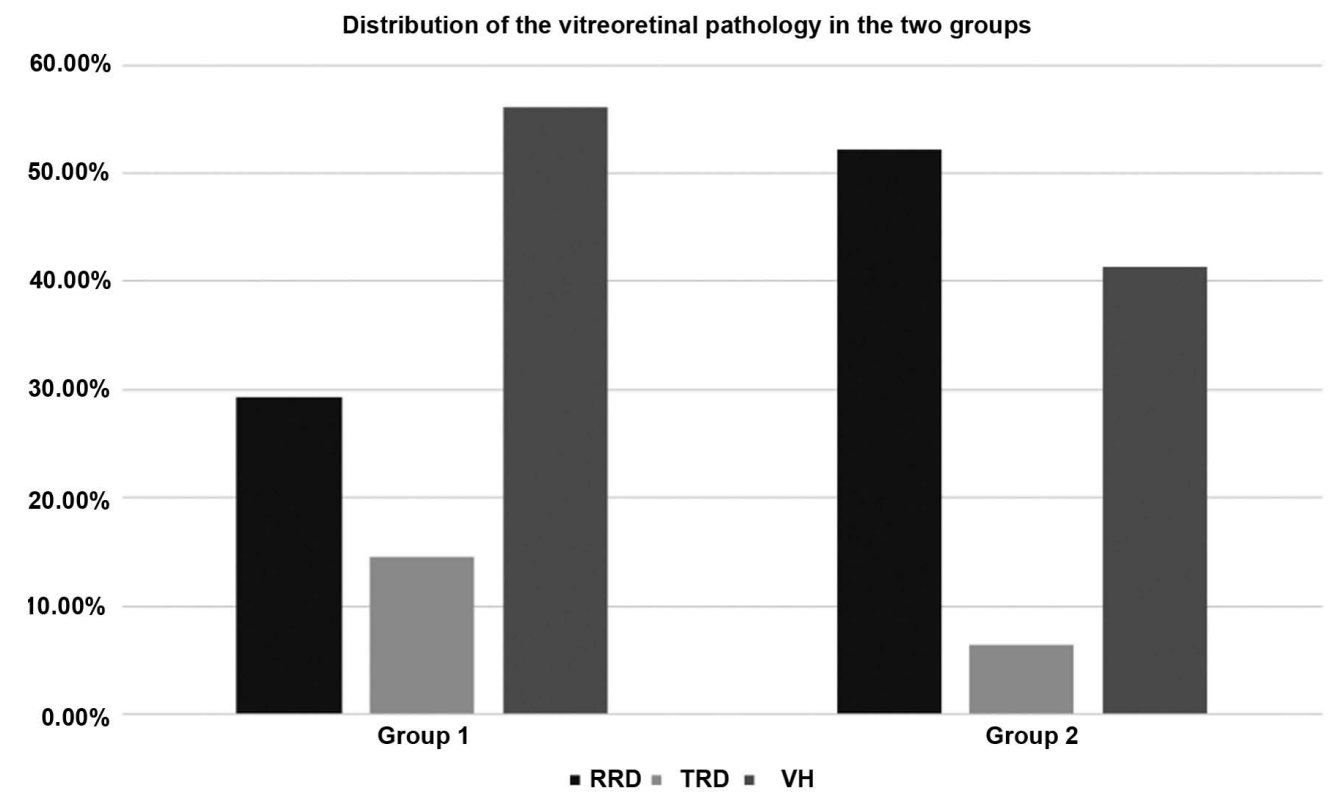

Figure 1. Percentage distribution of the vitreoretinal pathology in the two groups. RRD, rhegmatogenous retinal detachment; TRD, tractional retinal detachment; $\mathrm{VH}$, vitreous hemorrhage.

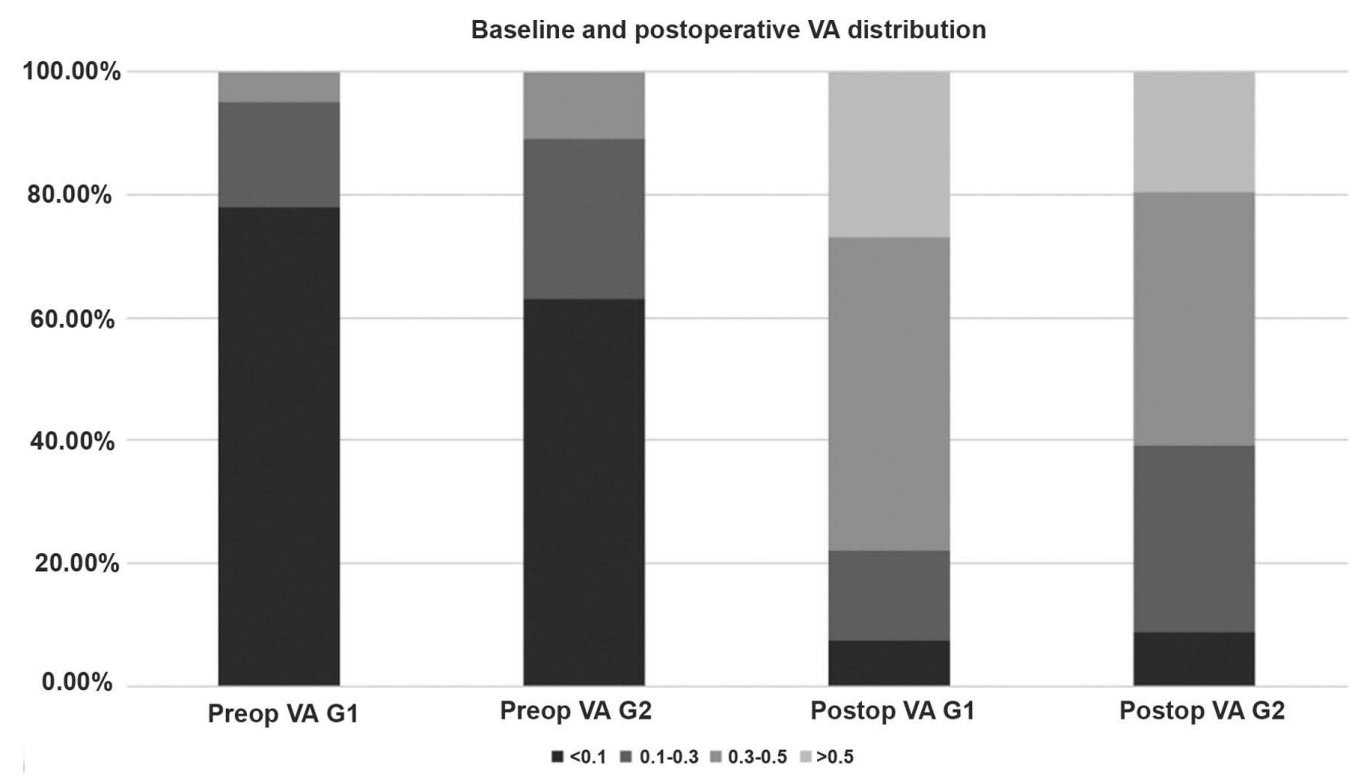

Figure 2. Baseline and postoperative VA distribution in both groups. VA, visual acuity.

6 months - 13 eyes (28.27\%). In the first postoperative day, $5(12.2 \%)$ eyes from group 1 had a mild fibrin reaction in the AC by comparison to $1(2.2 \%)$ in group 2 after PPV and $1(2.2 \%)$ after cataract surgery, which resolved with topical and subconjunctival injections of dexamethasone. There were no patients, in either group, with posterior synechia. Corneal edema was present in $17(41.46 \%)$ patients in group 1, $1(2.2 \%)$ patient from group 2 after PPV and 7 (15.2\%) patients from the same group after cataract surgery. In all cases, the corneal edema resolved during the first week postoperative with topical treatment.

The reoperation rate of all causes was $34.14 \%$ (14 eyes) in group 1 and $23.9 \%$ (11 eyes) in group 2 . Reoperation was due to: retinal redetachment in $9(21.95 \%)$ eyes from group 1 and in $8(17.4 \%)$ eyes from group 2; a new vitreous hemorrhage in $1(2.4 \%)$ patient from group 1 and $3(6.5 \%)$ from group 2 and displacement of the IOL in $4(9.75 \%)$ cases from group 1 .

The rate of posterior capsular opacification (PCO) encountered during the follow-up period was $17.07 \%$ ( 7 eyes) in group 1 and $10.87 \%$ (5 eyes) in group 2. Postoperative hypotony was registered in 6 eyes (14.63\%) from group 1 and 2 eyes (4.34\%) from group 2, after PPV, which resolved during the first week after surgery (Figs. 1-3).

\section{Discussion}

Performing cataract surgery after PPV may represent a challenge even for the most experienced surgeons. A vitrectomized 
Postoperative adverse reaction distribution

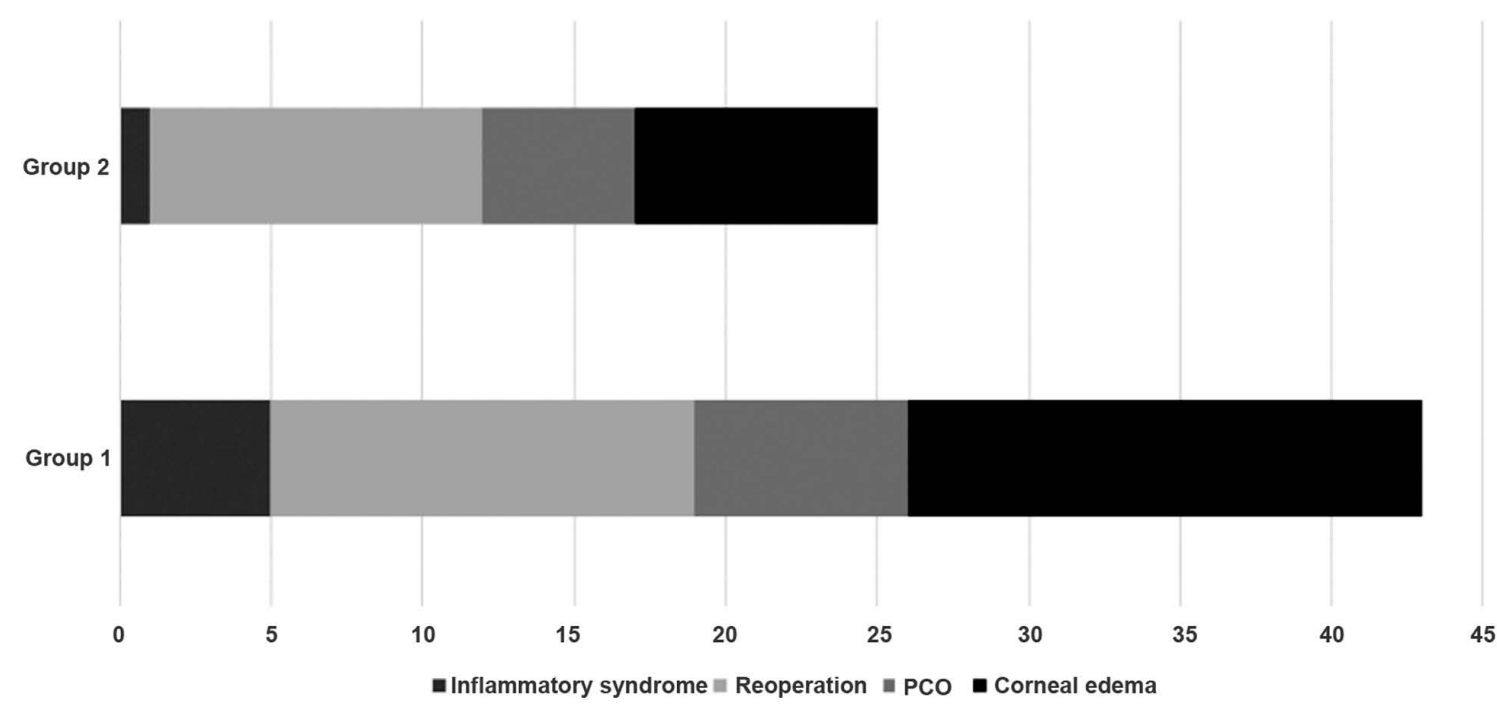

Figure 3. Postoperative adverse reaction distribution: Number of patients in each group presenting with postoperative inflammatory syndrome, posterior capsular opacification (PCO), corneal edema or in need of surgical reintervention.

eye presents with capsular damage and zonular weakness, a harder nucleus, unstable dynamics of the anterior chamber rendering the intervention more difficult than routine cases. The advantage of a combined procedure, besides the economic factors involved, is the clear eye media that allows a safer surgery, provides the ability to shave the vitreous base, especially during retinal detachment surgery, to recognize and treat a iatrogenic break and avoid the risk of touching the lens with the vitreoretinal instruments. In fact, cataract surgery cannot be avoided in most of the PPV patients, as Braunstein and Airiani (3) reported that $80 \%$ of these patients develop lens opacification during the first 2 years. Also, the combined procedure allows a quicker rehabilitation and socio-economical reintegration of the patient.

However, there are some shortcomings related to performing simultaneous surgeries. Besides a prolonged procedure, it is expected to have a higher ratio of anterior segment inflammation and synechia formation postoperative, by comparison to sequential surgery. There is a higher rate of capsular rupture and difficulty in accomplishing correctly the surgical steps due to depth variations of the anterior chamber, absence of red reflex or posterior displacement of the irido-lenticular diaphragm.

Analyzing the data obtained in this study, we observed that there are no major differences between the posterior capsular rupture rates between the two groups, nor between the intraoperative miosis rates. Both are higher in group 2, which benefited from sequential surgery, but there were more patients in this group. Miosis may be related to a variety of causes: included prolonged surgery, surgical trauma, but also previous medication administered to the patient (especially $\alpha$-blockers), pseudoexfoliation syndrome and in all cases responded well to intracameral epinephrine $0.001 \%$. There were more eyes with intraoperative posterior capsular rupture in group 2, $15.21 \%$ as compared with $7.31 \%$ in group 1 , most of them related to anterior chamber imbalance in silicone oil-filled eyes, or a weaker zonule in the vitrectomized eyes. Although the percentage of intraoperative posterior capsular rupture in the sequential surgery group is higher, the number of eyes affected by this incident was low, 3 in group 1 vs. 7 in group 2 .

Another concern while performing the combined procedure is the maintenance of the anterior chamber depth, which requires the use of a higher quantity of viscoelastics and in some cases the incomplete removal of the OVD at the end of the surgery. Also, IOL decentration, transfer of silicone oil or air/gas from the vitreous cavity to the anterior chamber and visualization issues during vitrectomy due to endothelial stria or the IOL margins may render the combined procedure more difficult.

Regarding the best time for IOL implantation in the combined procedure, there are some controversies. Some authors consider that it is preferable to inject the IOL at the end of the surgery, when both cataract and vitrectomy were resolved, thus avoiding the prismatic effect of the IOL edge that hampers the vitreous base vitrectomy (4). Other studies demonstrate that there is a lower risk of damaging the posterior capsule if the IOL is injected at the end of the cataract surgery and before PPV, the approach that was preferred in all cases included in group 1 in our study (5).

Simultaneous surgery is considered to have a higher risk of postoperative inflammation (6). Recent literature data reported rates of inflammatory reaction and fibrin formation in the anterior chamber after combined surgery comprised between $3.5-20 \%$ (7-9). The progress in instrumentation recorded during the last decade seems to have an important role in decreasing these rates. Earlier studies considered that the intact lens may act as a barrier, preventing the transfer of inflammatory factors between the anterior chamber and the vitreous cavity. In recent years, the levels of cytokines and growth factors were measured and reported at high values in the anterior chamber after cataract surgery and in the vitreous cavity after PPV $(10,11)$. Iris manipulation during anterior segment procedures presents an additional risk of inflammation with high levels of IL-1, IL-8, IL-0, IL-6 and TNF- $\alpha$, 
cytokines that are proven to be involved in the development of proliferative vitreoretinopathy (12). Also, in vitrectomized eyes, the inflammatory cytokines are present many months after PPV (13-17). These factors are known to promote fibrosis development under the form of proliferative vitreoretinopathy and posterior capsule opacification, which explains why there is a higher inflammatory risk when the procedures are accomplished simultaneously. In our study, the reoperation rate was higher in group 1, due to the occurrence of retinal redetachment in more eyes as compared with group 2, which can be explained by the breakdown of both the blood-aqueous barrier and the blood-retina barrier at the same time. The redetachment of the retina is also a factor that contributes additionally to the inflammatory cascade by releasing supplementary cytokines and pigment epithelium cells. When considering the percentage of reoperated patients due to redetachment, the values obtained in this retrospective study are comparable to literature data (18-20). However, some reinterventions were effectuated for reasons not related to the inflammatory process, such as IOL displacement or vitreous hemorrhage. One limitation of this study is the fact that we did not take into account the number of patients that needed reintervention due to secondary epiretinal membranes occurrence. In all cases, the anterior segment fibrinous reaction responded well to intensive local anti-inflammatory therapy, so this episode did not influence the final visual outcome.

The posterior capsule opacification rate was higher in group $1(17.07 \%)$ in comparison to group $2(10.87 \%)$. It is postulated that long-term endotamponade and inflammation contribute to a more frequent occurrence of $\mathrm{PCO}$ in these eyes (21). However, there is not a significant difference between the number of PCO cases in the two groups. Some eyes from group 2 suffered from an extended period of inflammation due to redetachment of the retina, hemorrhage or were operated for cataract while the endotamponade was in place.

The eyes included in group 1 were more prone to develop postoperative corneal edema, due to a prolonged and more complex surgical intervention. In both groups, the edema resolved during the first postoperative week with topical treatment. Nor the PCO rate, neither the corneal edema influenced the final BCVA, as in both groups it was comprised between 0.3 and 0.5 in the majority of the cases. The mean IOP values were higher in group 1 during the immediate postoperative period, but at the end of the follow-up time, the mean values were similar between the two groups. Postoperative inflammation and some difficulty in removing the viscoelastics from the eye during the combined procedure may have influenced this number. As regarding the effect of the silicone oil tamponade on IOP values, there was not a significant difference in the number of silicone oil-filled eyes in the two groups (16 from group 1 and 25 from group 2), nor in the period of endotamponade maintenance, which varied between 3 and 6 months.

In this retrospective study, we evaluated the surgical outcomes, possible complications and adverse reactions encountered after simultaneous cataract and posterior pars-plana vitrectomy in patients with coexisting pathologies. In conclusion, the combined procedure is safe and effective in obtaining a good morphological and functional result in this category of patients. Although the difficulty of the surgical procedure and the number of adverse reactions were higher in the group that underwent the simultaneous surgery, the rehabilitation was quicker for these patients and the final functional results were comparable between the two groups. The analysis of the risks and benefits of each procedure should be taken into account and the cases selected individually for either simultaneous or sequential surgery in order to obtain the best outcomes.

\section{Acknowledgements}

Not applicable.

\section{Funding}

No funding was received.

\section{Availability of data and materials}

The datasets used and/or analyzed during the current study are available from the corresponding author on reasonable request.

\section{Authors' contributions}

All authors contributed equally to the design of the study and participated in the preparation and the reviewing of the manuscript. DC, RLM and DCB contributed to the literature research and the analysis and critical interpretation of the data. ADM and DCB conceived the review and revised the manuscript. All authors read and approved the final version of the manuscript.

\section{Ethics approval and consent to participate}

The study was approved by the Ethics Committee of 'N. Oblu' Clinical Hospital (Iași, Romania) and informed consent was obtained from each patient.

\section{Patient consent for publication}

Not applicable.

\section{Competing interests}

All the authors declare that they have no competing interests.

\section{References}

1. El-Amir AN, Keenan TD, Abu-Bakra M, Tanner V, Yeates D and Goldacre MJ: Trends in rates of retinal surgery in England from 1968 to 2004: Studies of hospital statistics. Br J Ophthalmol 93: 1585-1590, 2009.

2. Ciobotaru OR, Lupu MN, Rebegea L, Ciobotaru OC, Duca OM, Tatu AL, Voinescu CD, Stoleriu G, Earar K and Miulescu M: Dexamethasone - chemical structure and mechanisms of action in prophylaxis of postoperative side effects. Rev Cchim Buchar 70: 843-847, 2019.

3. Braunstein RE and Airiani S: Cataract surgery results after pars plana vitrectomy. Curr Opin Ophthalmol 14: 150-154, 2003

4. Hurley C and Barry P: Combined endocapsular phacoemulsification, pars plana vitrectomy, and intraocular lens implantation. J Cataract Refract Surg 22: 462-466, 1996. 
5. Jain V, Kar D, Natarajan S, Shome D, Mehta H, Mehta H, Jayadev $\mathrm{C}$ and Borse N: Phacoemulsification and pars plana vitrectomy: A combined procedure. Indian J Ophthalmol 55: 203-206, 2007.

6. Aptel F, Colin C, Kaderli S, Deloche C, Bron AM, Stewart MW and Chiquet C; OSIRIS group: Management of postoperative inflammation after cataract and complex ocular surgeries: A systematic review and Delphi survey. Br J Ophthalmol 101: 1-10, 2017.

7. Wensheng L, Wu R, Wang X, Xu M, Sun G and Sun C: Clinical complications of combined phacoemulsification and vitrectomy for eyes with coexisting cataract and vitreoretinal diseases. Eur J Ophthalmol 19: 37-45, 2009.

8. Tosi GM, Balestrazzi A, Baiocchi S, Tarantello A, Cevenini G Marigliani D and Simi F: Complex retinal detachment in phakik patients: Previtrectomy phacoemulsification versus combined phacovitrectomy. Retina 37: 630-636, 2017.

9. Tayyab H, Khan AA and Javaid RMM: Clinical outcome of 23g Trans-conjunctival pars plana vitrectomy - a prospective comparison of Phaco-Vitrectomy with only vitrectomy in phakic eyes. Pak J Med Sci 33: 1123-1127, 2017.

10. Jakobsson G, Sundelin K, Zetterberg $H$ and Zetterberg $M$ : Increased levels of inflammatory immune mediators in vitreous from pseudophakic eyes. Invest Ophthalmol Vis Sci 56: 3407-3414, 2015

11. Iyengar L, Patkunanathan B, McAvoy JW and Lovicu FJ: Growth factors involved in aqueous humour-induced lens cell proliferation. Growth Factors 27: 50-62, 2009.

12. Moysidis SN, Thanos A and Vavvas DG: Mechanisms of inflammation in proliferative vitreoretinopathy: From bench to bedside. Mediators Inflamm 2012: 815937, 2012.

13. Gu R, Zhou M, Jiang C, Yu J and Xu G: Elevated concentration of cytokines in aqueous in post-vitrectomy eyes. Clin Exp Ophthalmol 44: 128-134, 2016.
14. Stanca HT, Petrović Z and Munteanu M: Transluminal Nd:YAG laser embolysis - a reasonable method to reperfuse occluded branch retinal arteries. Vojnosanit Pregl 71: 1072-1077, 2014.

15. Munteanu M, Rosca C and Stanca $\mathrm{H}$ : Sub-inner limiting membrane hemorrhage in a patient with Terson syndrome. Int Ophthalmol 39 461-464, 2019.

16. Stanca HT, Stanca S, Tabacaru B, Boruga M and Balta F: Bevacizumab in Wet AMD treatment: A tribute to the thirteen years of experience from the beginning of the anti-VEGF era in Romania. Exp Ther Med 18: 4993-5000, 2019.

17. Danielescu C, Stanca HT and Balta F: The management of lamellar macular holes: A review. Hindawi J Ophthalmol 2020: 3526316,2020

18. Loukovaara S and Haukka J: Repair of primary RRD - comparing pars plana vitrectomy procedure with combined phacovitrectomy with standard foldable intraocular lens implantation. Clin Ophthalmol 12: 1449-1457, 2018.

19. Maranduca MA, Branisteanu D, Serban DN, Branisteanu DC, Stoleriu G, Manolache N and Serban IL: Synthesis and physiological implications of melanic pigments. Oncol Lett 17: 4183-4187, 2019.

20. Park SP, Ahn JK and Lee GH: Morphologic changes in the anterior segment after phacovitrectomy for proliferative diabetic retinopathy. J Cataract Refract Surg 35: 868-873, 2009.

21. Jalil A, Steeples L, Subramani S, Bindra MS, Dhawahir-Scala F and Patton N: Microincision cataract surgery combined with vitrectomy: A case series. Eye (Lond) 28: 386-389, 2014. 